\title{
BCCN44.
}

\section{ON THE MEASUREMENT OF CROSS-SECTIONAL AREA OF NATURAL FIBERS}

\author{
Otavio da Fonseca Martins Gomes ${ }^{(1,2)}$, Felipe Pinheiro Teixeira ${ }^{(3)}$, Josimar Firmino de \\ Lima $^{(1)}$ and Flavio de Andrade Silva ${ }^{(3)}$ \\ (1) CETEM - Centre for Mineral Technology, Brazil \\ (2) Postgraduate Program in Geosciences, National Museum, Federal University of Rio de \\ Janeiro, Brazil
}

(3) Department of Civil and Environmental Engineering, Pontifícia Universidade Católica do Rio de Janeiro, Brazil

https://doi.org/10.21452/bccm4.2018.10.10

\begin{abstract}
The measurement of cross-sectional area of natural fibers is fundamental in the determination of their mechanical properties. Several authors investigated the mechanical behavior of natural fibers and reported, for samples of same species, different values for tensile strength and elastic modulus. This variability can be due to many factors, such as plant variability, damage during processing or cutting, testing conditions and accuracy. This work presents a method for the measurement of cross-sectional area natural fibers that guarantees the dimensional integrity of the fiber and in which the measurement is performed on a plane perpendicular to its longitudinal axis. It comprises sample preparation, image acquisition on a reflected light microscope, and image analysis using the open source software Fiji/ImageJ. The method was applied to curaua, hemp, and sisal samples.
\end{abstract}

Keywords: natural fibers, materials characterization, quantitative microscopy, mechanical behavior, mechanical properties

\section{INTRODUCTION}

Several authors investigated the mechanical behavior of natural fibers and reported very different values for tensile strength and elastic modulus for fibers of same species [1]. This variability can be due to any one or more of many factors, including fiber type, fiber variability (the position of fiber in the stem, fiber maturity or harvesting), fiber damage during its processing in the laboratory or industry, testing conditions and accuracy [2]. Luna and Lizarazo-Marriaga [3] pointed out that the cross-section area measurement must be carried out on a plane perpendicular to the longitudinal axis of the fiber and proposed a method to determine the cross-section area of Guadua angustifolia bamboo fibers. There are some papers 
in the literature [1,4-9] in which mechanical properties of natural fibers, like tensile strength and elastic modulus, were computed using cross-section measurements obtained through image analysis of SEM (scanning electron microscope) images. Besides not considering the slope of fibers with respect to the image plane, those papers do not take into account possible fibers crush and dismantling during cutting procedure and the fiber shrinking due to the high vacuum in the SEM.

The present work proposes a method that guarantees the dimensional integrity of the fiber and in which the measurement is performed on a plane perpendicular to its longitudinal axis.

\section{METHOD}

\subsection{Sample preparation}

The first preparation step is the treatment of fibers with hot water $\left(70^{\circ} \mathrm{C}\right)$ for $1 \mathrm{~h}$ to eliminate impurities retained on the fibers surface. Thereafter, the fibers are air dried for $48 \mathrm{~h}$.

A previously prepared epoxy resin block with $30 \mathrm{~mm}$ of diameter and $12 \mathrm{~mm}$ height is drilled with a $1.5 \mathrm{~mm}$ diameter drill to create 15 holes. The perforated block is placed on a Büchner funnel attached to a kitassato flask linked to a vacuum pump. A layer of filter paper between the Büchner funnel and the block separates it from the suction. Figure 1 shows this apparatus. It worth mentioning that it is possible to use a cheaper general-purpose epoxy resin to prepare the resin block to be perforated. The use of a high-cost epoxy resin for metallographic preparations, such as Struers Epofix, is required only for the fiber embedding in order to promote a good adhesion between fibers and surrounding resin and to mitigate the formation of bubbles.

One $10 \mathrm{~mm}$ length fiber sample is then carefully placed in each of the 15 holes of the block and subsequently the holes with fibers are filled with epoxy resin (Struers Epofix), as schematically illustrated in Figure 2. The vacuum is used to avoid fibers floating and to prevent the formation of bubbles during the $12 \mathrm{~h}$ of cold curing of resin.

After curing, the block is ground and polished in an automatic polishing machine (Struers Tegramin 20). Although, automatic polishing generally improves the quality of polished sections, it is not mandatory. Grinding and polishing tasks can be manually carried out with good results if they are carefully done. The grinding is carried out cooled with water using diamond impregnated metal discs with 125, 40, 9 and $6 \mu \mathrm{m}$ sized particles, during 3, 4, 4 and 10 min respectively. After grinding, the block undergoes an ultrasonic bath to remove any possible residues, to prevent scratching during polishing. The polishing procedure employs cloths with diamond suspensions of 3 and $1 \mu \mathrm{m}$ for approximately $20 \mathrm{~min}$ each, generating a highly polished section (Figure 3). 


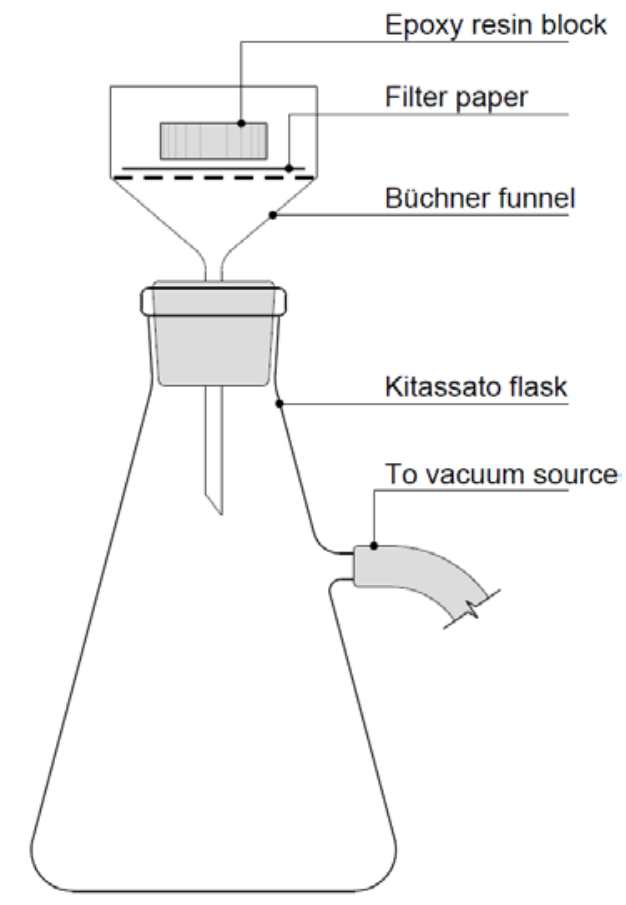

Figure 1: The resin embedding apparatus.

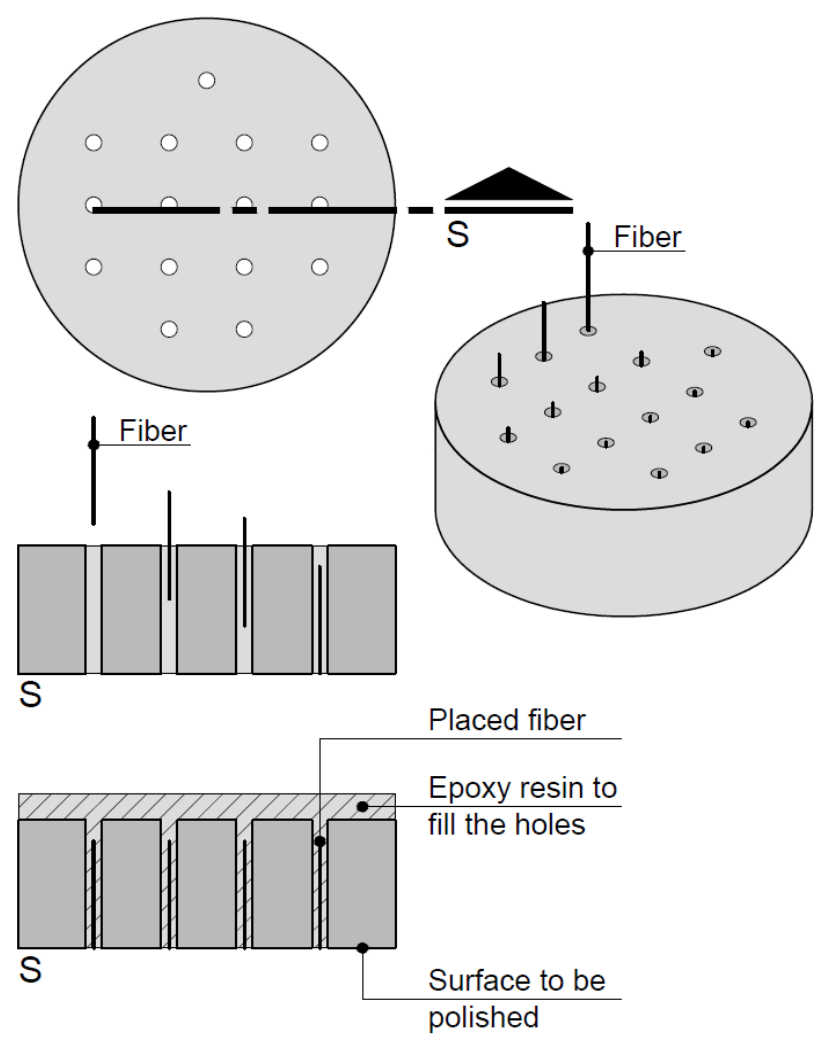

Figure 2: Schematic illustration of the block with 15 holes where fiber sample are placed and embedded with epoxy resin. 


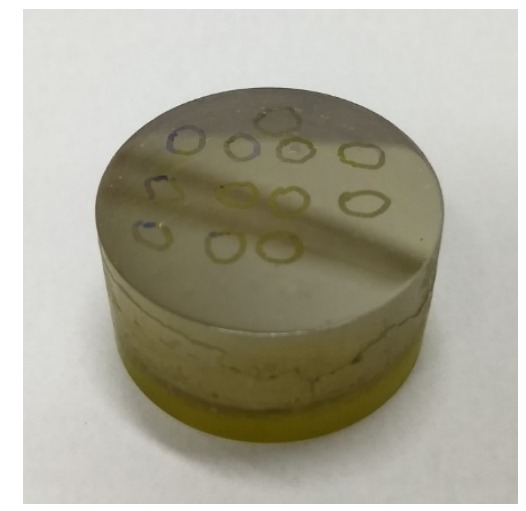

Figure 3: Block with fiber samples after curing and polishing. The fibers were circled with a marker pen to facilitate finding them at the microscope.

\subsection{Image acquisition}

A reflected light microscope with a digital camera (Zeiss Axioimager M2.m with Axiocam MRc) is used to generate an image of each fiber cross-section. Blank field contrast mode is employed. The magnification depends on each fiber type and plant species. Lamp voltage, exposition time and other operational parameters should be set to provide images with suitable brightness and contrast, avoiding intensity saturation in black or in white. Figure 4 shows images of fibers of: (a) sisal, (b) curaua, and (c) hemp, acquired using objective lenses of 20X, 50X, and 100X respectively.

(a)

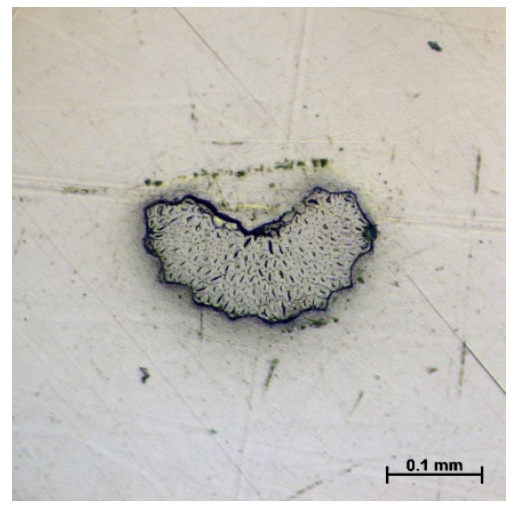

(b)

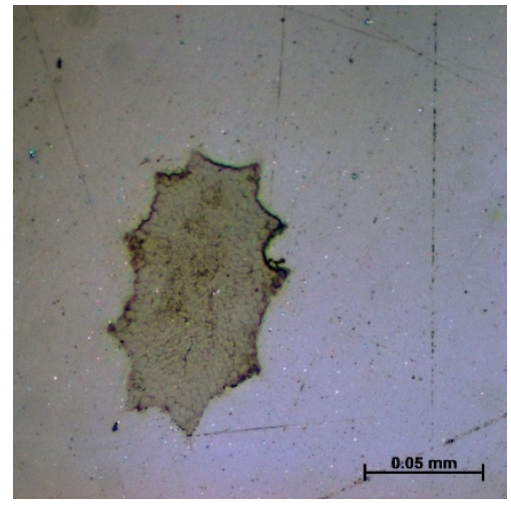

(c)

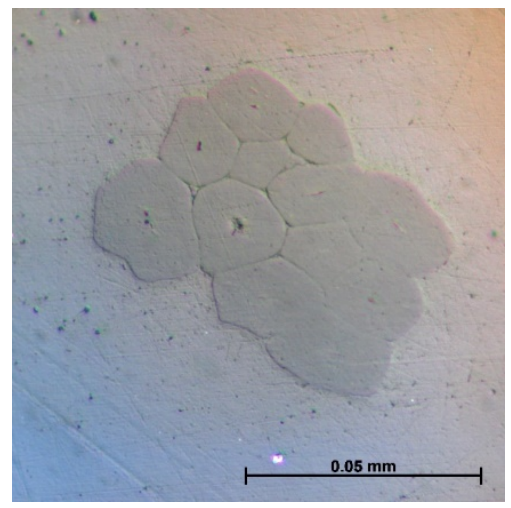

Figure 4: Images of fibers of: (a) sisal, (b) curaua, and (c) hemp, acquired using objective lenses of 20X, 50X, and 100X respectively.

\subsection{Image analysis}

The fiber cross-section is interactively segmented and then measured using Fiji/ImageJ. Fiji [10] is a free image processing package, a distribution of the popular open source software ImageJ [11] bundling a lot of plugins and different scripting languages.

At least before analyzing the first image, the scale (in microns/pixel, for instance) and the desired measurements (area, in the present case) must be set. This set-up can be made interactively accessing the functions Set Scale and Set Measurements in the Analyze menu or it can be done through the macro routine shown in Figure 5. 


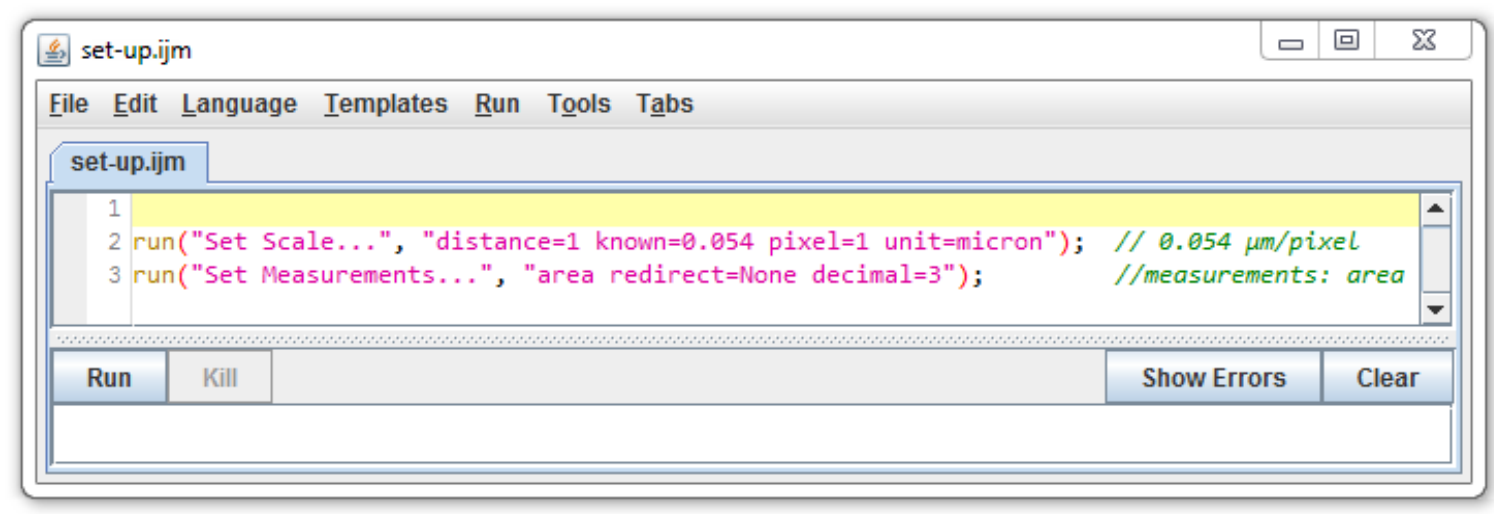

Figure 5: Macro routine to set scale and measurements.

The image analysis procedure follows this sequence:

(a) Pencil Tool is used to carefully freehand outline the fiber cross-section;

(b) Wand Tool (with parameters Tolerance $=0$ and Mode $=$ Legacy, which can be accessed through a double-click on the tool icon) is employed to create a selection from a click in the drawn outline;

(c) Measure (in Analyze menu) performs the previously set measurements in the outlined region.

Figure 6 presents the analysis of a fiber showing from top to bottom: the Fiji main window, the image of an outlined hemp fiber, and the results window with the obtained area value in $\mu \mathrm{m}^{2}$.

In the image analysis field, segmentation is the technical term used for the discrimination of objects from the image background and among themselves. It is generally the critical step of an image analysis routine, since the measurements are done on the segmented objects. Segmentation is a complex task that tries to represent computationally a cognitive process that is inherent to the human vision. When we look at an image we use many different inputs to distinguish the objects, such as color, boundaries, shapes, textures, among others. Our brains process this information in parallel at high speed, using previous experience. Computers, on the other hand, do not have the same associative power yet [12]. Although we can easily recognize the cross-section of a natural fiber in an image obtained as described in the proposed method, its automatic segmentation is not easy. Natural fibers are non-opaque materials, as well as the epoxy resin, hence they present a poor contrast in images from reflected light microscopy. Moreover, natural fibers display a wide variability of size and shape, and their cross-sections present void lumens and lumens filled with resin. Therefore, we have opted to employ an interactive segmentation procedure in the proposed method. 

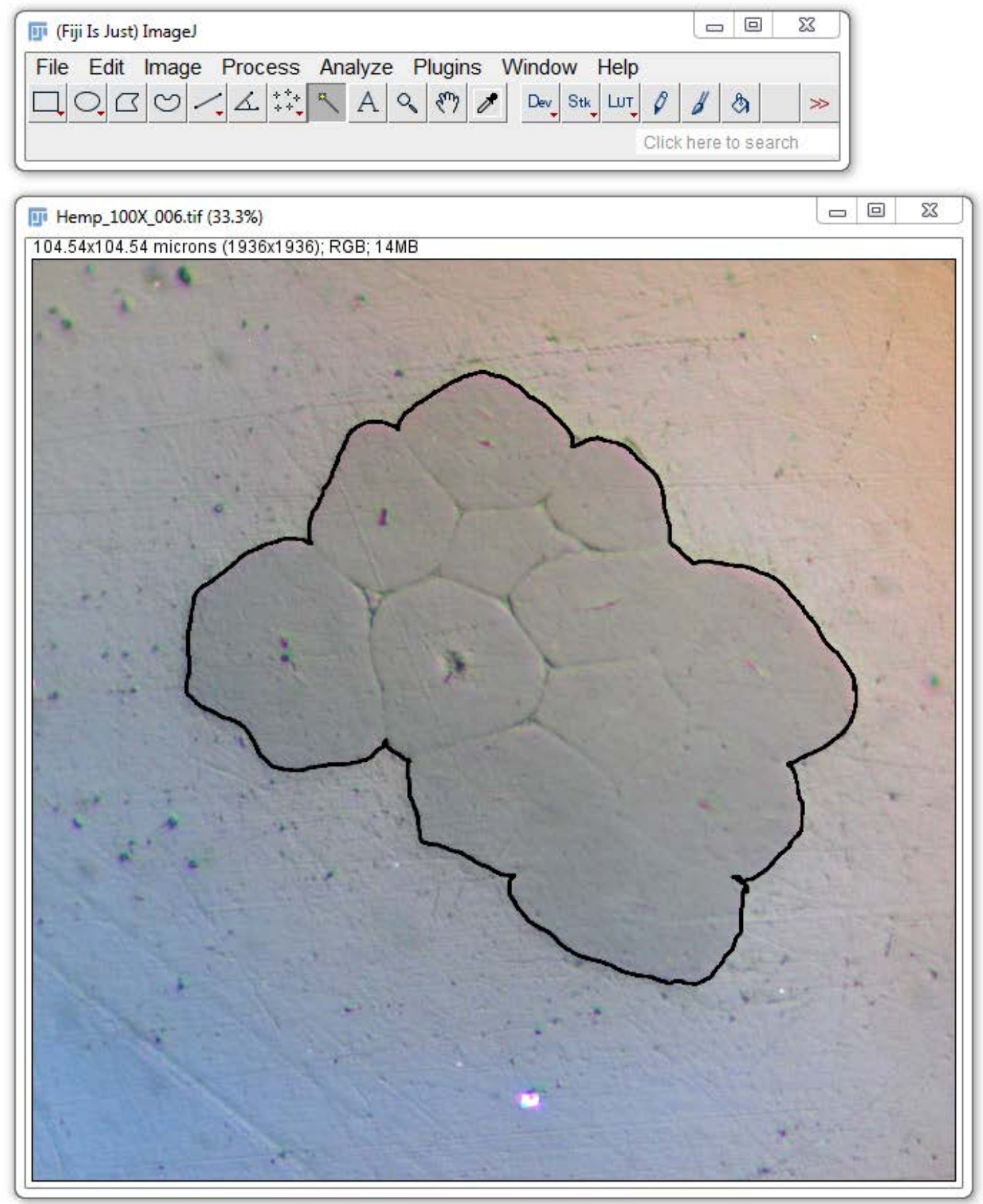

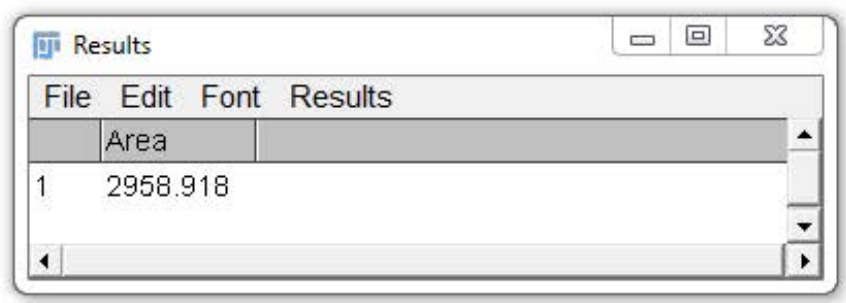

Figure 6: The analysis of a fiber, showing from top to bottom: the Fiji main window, the image of an outlined hemp fiber, and the results window with the obtained area value in $\mu \mathrm{m}^{2}$.

The use of a SEM instead of a reflected light microscope can provide better images of natural fibers cross-sections that allow the automation of their image analysis. In back-scattered electrons images of polished (plane) specimens, materials present grey levels proportional to 
their average atomic numbers [12]. Thus, in such kind of image natural fibers can be easily discriminated from surrounding resin through their different grey levels by an automatic segmentation procedure. Nevertheless, besides its high cost, the use of a SEM has other disadvantages. An additional preparation step is required, the polished block should be coated (for instance, with evaporated carbon) to become conductive so that the SEM can provide high quality images. Furthermore, due to the high vacuum in the coater and in the SEM, natural fibers may shrink and detach from the resin, and their lumens may open, as one can see comparing the images in Figure 7 that shows the cross-section of a sisal fiber imaged by: (a) reflected light microscopy using the objective lens of 50X, and (b) SEM with the same optical resolution.

(a)

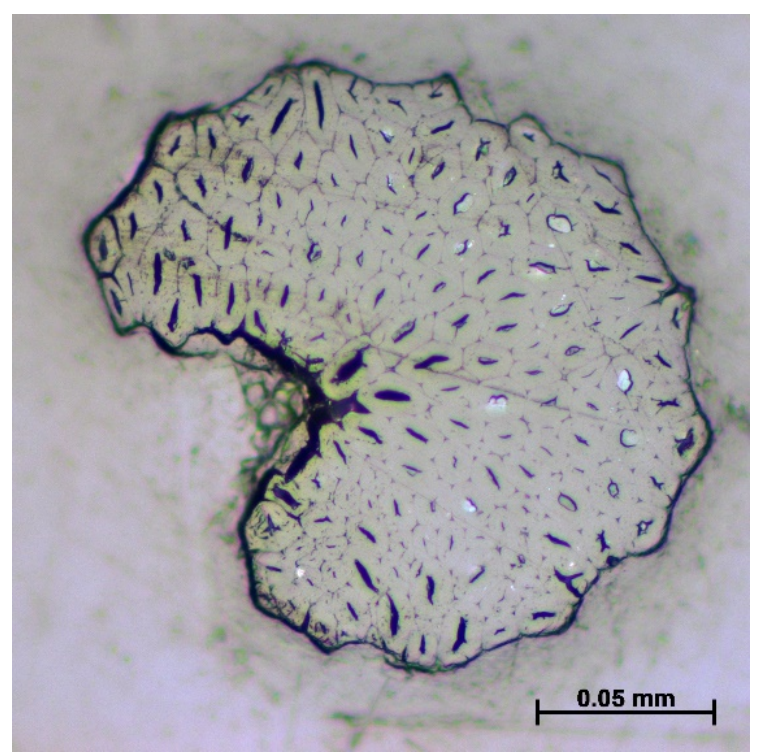

(b)

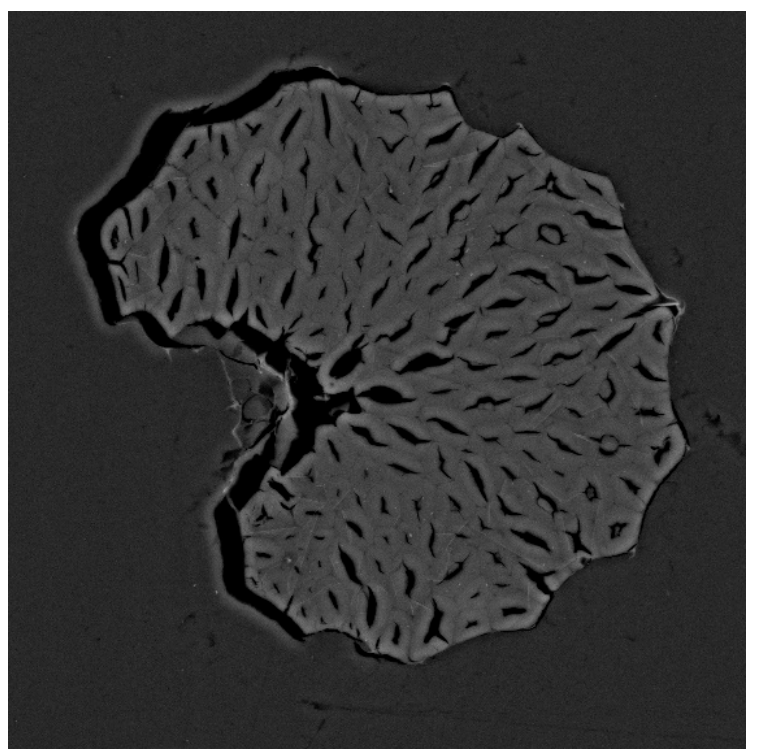

Figure 7: Cross-section of a sisal fiber imaged by: (a) reflected light microscopy (objective lens of 50X), and (b) SEM.

\section{CONCLUSIONS}

This work presents a method for the measurement of cross-sectional area natural fibers that comprises sample preparation, image acquisition on a reflected light microscope, and interactive image analysis using the open source software Fiji/ImageJ. The sample preparation procedure guarantees a clean perpendicular cut. The use a reflected light microscope avoids the fiber shrinking due to the high vacuum in the SEM.

Future works can focus on obtaining images through different microscopy techniques in order to provide images with a suitable contrast to allow natural fibers cross-section measurements through an automatic image analysis routine. Moreover, the image acquisition step may also be automatized, since the holes positions in the perforated block are known and the fibers can be located into them through image analysis.

\section{ACKNOWLEDGEMENTS}

The authors acknowledge the support from the Brazilian funding agencies CNPq, CAPES, FINEP, and FAPERJ. F.P. Teixeira thanks CAPES for the D.Sc. candidate scholarship number 88887.165362/2018-00. 


\section{REFERENCES}

[1] Fidelis, M.E.A., Pereira, T.V.C., Gomes, O.D.M., Silva, F.A., Toledo-Filho, R.D., 'The effect of fiber morphology on the tensile strength of natural fibers', J. Mater. Res. Technol. 2 (2) (2013) 149-157.

[2] Hughes, M., 'Defects in natural fibres: their origin, characteristics and implications for natural fibre-reinforced composites', J. Mat. Sci. 47 (2) (2012) 599-609.

[3] Luna, P., Lizarazo-Marriaga, J., 'Proposed Method for Determining Cross-Sectional Area of Guadua angustifolia Bamboo Fibers', J. Nat. Fibers (2017).

[4] Silva, F.A., Chawla, N., Toledo-Filho, R.D., 'Tensile behavior of high performance natural (sisal) fibers', Compos. Sci. Technol. 68 (15-16) (2008) 3438-3443.

[5] Ramirez, F., Maldonado, A., Correal, J.F., Estrada, M., 'Bamboo-Guadua angustifolia kunt fibers for green composites', in Proceedings of 18th International Conference on Composite Materials, 2011 (The Korean Society of Composite Materials), 1-4.

[6] Diaz, J.P.V., Silva, F.A., D’Almeida, J.R.M., 'Effect of Peach Palm Fiber Microstructure on its Tensile Behavior', BioResources 11 (4) (2016) 10140-10157.

[7] Ferreira, S.R., Silva, F.A., Lima, P.R.L., Toledo-Filho, R.D., 'Effect of hornification on the structure, tensile behavior and fiber matrix bond of sisal, jute and curauá fiber cement based composite systems', Construction and Building Materials 139 (2017) 551-561.

[8] Ferreira, S.R., Pepe, M., Martinelli, E., Silva, F.A., Toledo-Filho, R.D., 'Influence of natural fibers characteristics on the interface mechanics with cement-based matrices', Composites Part B: Eng. 140 (2018) 183-196.

[9] Joshi, A., Mangal, R., Bhojak, N., 'Study of the mechanical properties of Ziziphus nummularia (ber) fibers for formation of fiber reinforced composites', AIP Conference Proceedings 1953 (2018).

[10] Schindelin, J., Arganda-Carreras, I., Frise, E., Kaynig, V., Longair, M., Pietzsch, T., Preibisch, S., Rueden, C., Saalfeld, S., Schmid, B., Tinevez, J.Y., White, D.J., Hartenstein, V., Eliceiri, K., Tomancak, P., Cardona, A., 'Fiji: an open-source platform for biological-image analysis', Nat. Methods 9 (7) (2012) 676-682.

[11] Schneider, C.A., Rasband, W.S., Eliceiri, K.W., 'NIH Image to ImageJ: 25 years of image analysis', Nat. Methods 9 (7) (2012) 671-675.

[12] Gomes, O.D.M., Paciornik, S., 'Multimodal Microscopy for Ore Characterization', in Kazmiruk, V. (ed.), 'Scanning Electron Microscopy' (InTech, Rijeka, 2012) 313-334.

[13] Goldstein, J.I., Newbury, D.E., Echlin, P., Joy, D.C., Lyman, C.E., Fiori, C., Lifshin, E., Sawyer, L., Michael, J.R., 'Scanning Electron Microscopy and X-ray Microanalysis', 3rd Edn (Springer, New York, 2003). 